\title{
Methods for Modeling and Predicting Mechanical Deformations of the Breast Under External Perturbations
}

\author{
Fred S. Azar ${ }^{1}$, Dimitris N. Metaxas ${ }^{2}$, Mitchell D. Schnall ${ }^{3}$ \\ ${ }^{1}$ Dept. of BioEngineering, University of Pennsylvania (fredazar@seas .upenn.edu) \\ ${ }^{2}$ Dept. of Computer Science, University of Pennsylvania \\ (dnm@graphics.cis.upenn.edu) \\ ${ }^{3}$ Dept of Radiology, University of Pennsylvania (schnall@oasis.rad.upenn.edu)
}

\begin{abstract}
Currently, High Field (1.5T) Superconducting MR imaging does not allow live guidance during needle breast procedures, which allow only to calculate approximately the location of a cancerous tumor in the patient breast before inserting the needle. It can then become relatively uncertain that the tissue specimen removed during the biopsy actually belongs to the lesion of interest. A new method for guiding clinical breast biopsy is presented, based on a deformable finite element model of the breast. The geometry of the model is constructed from MR data, and its mechanical properties are modeled using a non-linear material model. This method allows imaging the breast without or with mild compression before the procedure, then compressing the breast and using the finite element model to predict the tumor's position during the procedure in a total time of less than a half-hour.
\end{abstract}

\section{Introduction}

The ability to identify a mass in the breast requires the mass to have a different appearance (or a different contrast) from normal tissue. With high-field MRI, the contrast between soft tissues in the breast is 10 to 100 times greater than that obtained with X-rays [1]. A MR image-guided breast localization and biopsy system allows the differentiation between the benign enhancing lesions, and carcinomas [1]. However, the appearance, size and shape of the potential cancer lesion greatly depend on dynamics of the contrast-enhancing agent. Furthermore, the needle is not a very sharp object, and cannot be inserted smoothly in the breast, causing unwanted deformations. The breast could be compressed so as to minimize internal deformations, however that would cause blood to be squeezed out and could dramatically alter the appearance and shape of the lesion on the MR image.

The above limitations coupled with the deformable structure of the breast make needle procedures very sensitive to the initial placement of the needle, and to the amount of breast compression. It thus becomes relatively uncertain that the tissue specimen removed during the biopsy procedure actually belongs to the lesion of interest, due to the added difficulty of accurately locating the tumor's boundaries inside the breast.

We present a virtual deformable breast model of the patient, based on MR data, and using non-linear material models. This model can be used effectively in a new method for guiding clinical breast biopsy. This method involves imaging the patient's breast without any or little compression before a needle procedure, then compressing the breast, and its virtual finite element model (by applying the same pressure to W. Niessen and M. Viergever (Eds.): MICCAI 2001, LNCS 2208, pp. 1267-1270, 2001.

(C) Springer-Verlag Berlin Heidelberg 2001 
both), and using the displacement of the virtual tumor model to predict the displacement of the real cancer tumor. Other possible applications include registration of data sets from different imaging modalities, diagnosis, measurements, surgery planning, simulations of deformation due to inserting a needle, and further away, virtual surgery, and tele-surgery.

\section{Methods}

We had presented in [2] an initial attempt to model a patient breast by developing a preliminary FE model of the breast. We extend that methodology to allow clinical use of the model by :

1. Improving the technique. A new meshing algorithm is used to allow variable meshing density in the model, in which the mesh density is increased around the point of interest, and decreased away from it. A new material model for fatty tissue is introduced following the rationale presented in [2]. The non-linear elastic modulus of fatty tissue $E_{f a t}$ is a function of the strain $\varepsilon_{f a t}$ :

$$
E_{f a t}\left(\varepsilon_{f a t}\right)=A \cdot \varepsilon_{f a t}^{2}+B \cdot \varepsilon_{\text {fat }}+C
$$

where $\mathrm{A}, \mathrm{B}$ and $\mathrm{C}$ are determined based on the boundary conditions. A detailed description of the derivation of this equation can be found in [3]. Finally, a faster time-convergence algorithm employing an Euler method with adaptive step sizing is used. The improvements can reduce the computation time to less than ten minutes.

2. Validating the method. A deformable silicon gel phantom was built to study the movement of a stiff inclusion inside a deformable environment under plate compression [4]. The geometry of the deformable phantom consists of a rectangular box $(84 \times 82 \times 70 \mathrm{~mm})$ containing a rectangular inclusion $(20 \times 23 \times 20 \mathrm{~mm})$, which is 4.3 times stiffer than the surrounding silicone. The phantom was imaged undeformed, then compressed $14 \%(9.8 \mathrm{~mm})$. The performance of our software algorithm was compared to that of a commercial FEM software package ABAQUS, by measuring the displacement vectors of the 8 corners of the stiff inclusion and its center.

3. Testing the model and its performance on a patient. A patient's breast was imaged uncompressed and then compressed $26 \%$. The corresponding deformable model was built, and was virtually compressed to match the real compression amount. We tracked the displacement of a small cyst and of two vitamin E pills taped to the superior and inferior parts of the breast. We also tested the model through a convergence analysis and a sensitivity analysis (in which the material parameters were varied over a physiologically relevant range, within two standard deviations of their experimentally derived average value) [3].

\section{Results and Discussion}

\subsection{Silicone Phantom Experiment}

The results (Fig. 1) show that all of the average displacement errors per node between the two simulations are under $1 \mathrm{~mm}$, and that using a small strain approximation [2] instead of the Lagrangian finite strain expression, in our finite element algorithm does not introduce a significant error in simulations of large deformation. 

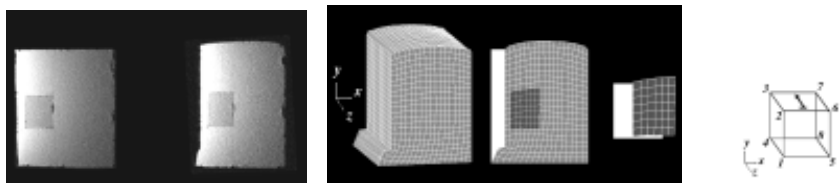

Fig. 1. Uncompressed and compressed axial MR slice of phantom (left), 3D view of model including axial slice through center of inclusion and axial view of inclusion center, before and after compression (center)

\subsection{Patient Breast Compression Experiment}

The MR images containing the landmarks and the corresponding model slices are shown in Fig.2. The arrows in the figure indicate the location of the landmarks.

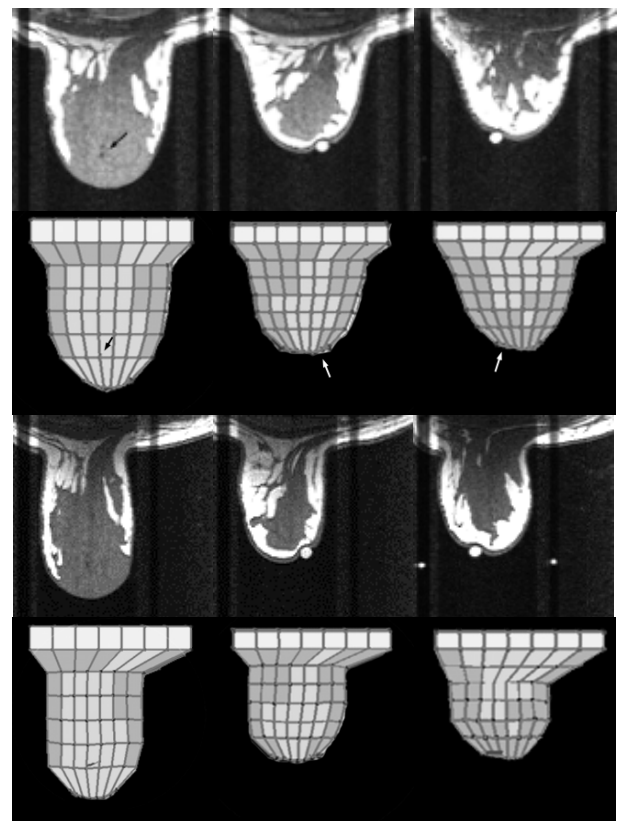

Fig. 2. Uncompressed MR images (upper left), uncompressed model slices (upper right), compressed MR slices (lower left), and corresponding virtually compressed model slices (lower right) after virtual compression

All of the simulations in the variational studies were done on a SGI Octane Workstation with $2195 \mathrm{MHz}$ IP30 processors (MIPS R10000 processors), and 256 Megabytes of memory (RAM). The model contains 512 nodes, 343 3D hexahedral elements, and 294 2D triangular elements. Table 1 shows the displacement differences (Model-Real) of the landmarks' center of gravity, and the $\%$ Misclassification which compares the number of misclassified pixels in the model with the compressed MRI. 
Table 1. Averages and standard deviations (in parentheses) of sensitivity analysis

\begin{tabular}{|l|l|l|l|}
\hline & Cyst & Vit. E pill (INF) & Vit. E pill (SUP) \\
\hline Disp. Difference (mm) & $1.3(\mathbf{0 . 1})$ & $3.5(\mathbf{0 . 1})$ & $4.6(\mathbf{0 . 1})$ \\
\hline \%Misclassification & $11.8(\mathbf{1 . 0})$ & $25.1(\mathbf{1 . 2})$ & $31.4(\mathbf{0 . 5})$ \\
\hline
\end{tabular}

The convergence analysis shows that the simulation indeed converges to the solution, as the model mesh is refined. The material properties sensitivity analysis (Table 1) shows that large variations in material properties parameters do not significantly affect the parameter results.

\section{Conclusion}

The final results show that it is possible to create a non-linear deformable model of the breast capable of modeling and predicting deformations in less than a half-hour : the average times to completion were 12 minutes for segmentation of MR data, 3 minutes for the model mesh creation, and 14 minutes for the model simulation.

\section{References}

1. Orel SG, Schnall MD et al:: MR Imaging-guided Localization and Biopsy of Breast Lesions: Initial Experience. Radiology, Vol. 193 (1994) 97-102

2. Azar FS, Metaxas DN, Schnall MD: A Finite Element Model of the Breast for Predicting Mechanical Deformations during Biopsy Procedures. IEEE Workshop, Mathematical Methods in Biomedical Image Analysis (2000) 38-45

3. Azar FS, Metaxas DN, Schnall MD: A Deformable Finite Element Model of the Breast for Predicting Mechanical Deformations under External Perturbations. J. Acad. Radiology (2001)

4. Azar FS, Metaxas DN, Miller RT, Schnall MD: Methods for Predicting Mechanical Deformations in the Breast during Clinical Breast Biopsy. 26th IEEE Annual N.E. BioEngineering Conference (2000) 